\title{
AÇÃO DE UM REGULADOR DE CRESCIMENTO NO ENRAIZAMENTO DE ESTACAS DE QUATRO PLANTAS ORNAMENTAIS *
}

\author{
Pedro D. Fernandes ** \\ Paulo R. C. Castro *** \\ SÉrgio N. KRONKA \\ IVor B. Aguiar **
}

\section{RESUMO}

\begin{abstract}
Estudou-se o efeito da aplicação, pelo método lento, de um regulador de crescimento, sob três diferentes concentrações, na indução de raizes em estacas de Rhododendron Simsii, Cupressus sempervirens, Bougainvilleu spectabilis e Thuya occidentalis.

Pela análise do número de estacas enraizadas verificou-se, que, pela ordem, os melhores resultados foram obtidos com Cupressus, Rhododendron, e finalmente Bougainvillea ou Thuya. Para R. Simsii a dosagem de $10 \mathrm{ml}$ do regulador de crescimento por litro de água, mostrou ser mais adequada; sendo que para C. sempervirens revelou ser de $20 \mathrm{ml} / 1$ a dose mais indicada. No caso de $\mathbf{B}$. spectabilis, a dosagem de $10 \mathrm{ml} / 1$ foi a mais favorável; sendo que a de $20 \mathrm{ml} / 1$ foi mais promissora no enraizamento de $\mathbf{T}$. occidentalis.

$\mathrm{Na}$ análise do peso fresco médio das raízes, revelaram-se, pela ordem, resultados mais favoráveis para Cupressus, Rhododendron ou Bougainvillea e finalmente Thuya. A dosagem de $20 \mathrm{ml} / 1$ mostrou-se mais adequada para $R$. Simsii, $C$. sempervirens e $\mathbf{T}$. occidentalis; sendo que para B. spectabilis as doses de 10 ou $20 \mathrm{ml} / 1$ mais favoráveis, revelaram-se significativamente distintas do controle $(0 \mathrm{ml} / \mathrm{l})$.
\end{abstract}

\section{INTRODUÇÃO}

A formação de raízes em estacas depende das condições internas da planta de origem e das condições ambientais em que são colocadas. Estacas oriundas de muitas espécies vegetais apresentam fácil enraizamento sob condições adequadas, outras porém necessitam de tratamento com substâncias indutoras de raízes para sua propagação vegetativa (THIMANN \& BEHNKE, 1950).

- Entregue para publicação em 13/11/1973.

* Departamento de Fitotecnia. F. M. V. A. Jaboticabal - CESESP.

** Departamento de Botánica. E. S. A. "Luiz de Queiroz" - U. S. P.

**** Departamento de Ciências Físicas e Matemáticas. F. M. V. A. Jaboticabal - CESESP. 
ZIMMERMAN et al. (1933) verificaram pela primeira vez que o monóxido de carbono possuia uma capacidade marcante e específica de promover 0 enraizamento de estacas.

FISCHNICH (1935) verificou que o ácido indolacético, quando aplicado corretamente, podia estimular a produção de raizes em plantas intactas. COOPER (1935) demonstrou que a aplicação do ácido indolacético em pasta de lanolina podia ser utilizada com sucesso no enraizamento de estacas.

Novas técnicas de propagação vegetativa foram desenvolvidas com a descoberta de diversos reguladores de crescimento sintéticos (ZIMMERMAN \& WILCOXON, 1935) com capacidade de promover a formação de raizes. Sais potássicos e ésteres do ácido indolbutírico, ácido naftalenacético e ácido indolacético são utilizados rotineiramente no tratamento de estacas para promover enraizamento. Algumas espécies responderam melhor para um ou mais destes compostos do que para outros, sendo que a utilização de dosagens corretas do regulador de crescimento é importante, pois as concentrações ótimas variam com as espécies estudadas (KRAMER \& KOZLOWSKI, 1960). A concentração ideal de reguladores de crescimento para aplicação em solução tem sido determinada para o enraizamento de muitas plantas, sendo que diversos produtos comerciais são hoje disponíveis.

Após a base da estaca ser tratada com o regulador de crescimento indutor de raízes, os carboidratos são translocados para a área tratada, aumenta a taxa de respiração e ocorrem transformações nos carboidratos e nos compostos nitrogenados orgânicos. $O$ regulador de crescimento pode acelerar o metabolismo normal e aumentar o número de primórdios radiculares (SNYDER, 1954).

RUBIA et al. (1965) obtiveram maior porcentagem de estacas enraizadas de amoreira (Morus alba cv. 'Catania' 1), com aplicação do ácido betaindolacético na concentração de $100 \mathrm{ppm}$; sendo que BRANDI \& BARROS (1971) verificaram uma tendência no aumento do número de estacas enraizadas de Pinus caribaea var. hondurensis, devido à aplicação dos ácidos indolpropiônico e indolbutírico, apesar de não terem sido constatadas diferencas significativas.

ARENS \& ARENS (1971) consideram que não se obtiveram resultados promissores no enraizamento de estacas de Eucalypłus, pela aplicação de hormônios de crescimento nas condições do Estado de São Paulo, porque sua utilização em estacas em que não existem reservas acumuladas não é capaz de estimular a produção de raízes adventíceas.

O objetivo deste trabalho foi verificar o efeito da aplicação de diferentes concentrações de um regulador de crescimento (Exubérone - Produto comercial da Société des Usines Chimiques Rhône-Poulenc da França, gentilmente oferecido pela Rhodia do Brasil) capaz de induzir o enraizamento, em estacas de quatro plantas ornamentais. 


\section{MATERIAIS E MÉTODOS}

Neste experimento utilizaram-se estacas uniformes de quatro espécies ornamentais, Rhododendron Simsii, Cupressus sempervirens, Bougainvillea spectabilis e Thuya occidentalis. Coletaram-se essas estacas, com $15 \mathrm{~cm}$ de comprimento, para plantio, em 14 de outubro de 1972. Durante o período de tempo que antecedeu ao plantio, conservou-se a extremidade basal das estacas imersa em água, com a finalidade de manter o processo de absorção, prevenindo o dessecamento das mesmas.

Posteriormente tratou-se as estacas com soluções de Exubérone, nas dosagens de 0,10 e $20 \mathrm{ml} /$ litro de água.

Ef etuou-se o tratamento lento das estacas, mantendo-se uma altura de 2,5 $\mathrm{cm}$ da base das mesmas imersa por 24 horas nas soluções aquosas do composto líquido promotor de enraizamento. Antes do plantio em recipientes de polietileno $(15 \times 28 \mathrm{~cm})$, tendo como substrato solo arenoso, realizou-se a lavagem da extremidade basal das estacas em água corrente.

O delineamento experimental utilizado foi em blocos casualizados (SNEDECOR, 1966; GOMES, 1970) no esquema fatorial $4 \times 3$, com 4 espécies vege-tais de diferentes gêneros e 3 dosagens distintas do produto regulador de crescimento.

O número total de parcelas foi de 48; sendo que cada uma possuia 15 estacas, perfazendo um total de 720 estacas.

As espécies vegetais utilizadas foram, Rhododendron Simsii (E1),Cupressus sempervirens (E2), Bougainvillea spectabilis (E3) e Thuya occidentalis (E4). As dosagens de Exubérone aplicadas foram de $0 \mathrm{ml} / 1$ (D1), $10 \mathrm{ml} / 1$ (D2) e $20 \mathrm{ml} / \mathrm{l}$ (D3).

A retirada das amostras para a determinação do número de estacas enraizadas e do peso fresco médio das raízes, efetuou-se 80 dias após o plantio, em 2 de janeiro de 1973.

Para a análise de variância dos dados da porcentagem de pegamento das estacas, os resultados foram transformados para arc sen $\sqrt{\mathrm{p}} / 100$. De acordo com STEEL \& TORRIE (1960), nas parcelas em que as porcentagens foram de $0 \%$ ou $100 \%$, utilizaram-se respectivamente das expressões $1 / 4 \mathrm{n}$ e $100-1 / 4 \mathrm{n}$, sendo $\mathrm{n}$ o número de estacas iniciais.

\section{RESULTADOS E DISCUSSAOO}

Os resultados obtidos da contagem do número de estacas enraizadas por parcela eneontram-se no quadro 1 . 
Quadro 1 - Número de estacas enraizadas por parcela.

\begin{tabular}{l|c|c|c|c}
\hline Tratamentos & 19 Bloco & 20 Bloco & 39 Bloco & 49 Bloco \\
\hline E1D1 & 2 & 2 & 1 & 2 \\
E1D2 & 6 & 8 & 8 & 7 \\
E1D3 & 4 & 4 & 4 & 3 \\
E2D1 & 2 & 1 & 0 & 2 \\
E2D2 & 8 & 8 & 10 & 9 \\
E2D3 & 12 & 13 & 15 & 15 \\
E3D1 & 1 & 0 & 0 & 0 \\
E3D2 & 5 & 4 & 4 & 4 \\
E3D3 & 2 & 1 & 2 & 2 \\
E4D1 & 1 & 1 & 1 & 1 \\
E4D2 & 0 & 0 & 0 & 0 \\
E4D3 & 7 & 6 & 5 & 7 \\
\hline
\end{tabular}

Estes resultados foram transformados para arc sen $\sqrt{\mathrm{p} / 100}$, e a análise de variância, de acordo com o esquema fatorial, encontra-se no quadro 2.

Quadro 2 - Análise da variância do número de estacas enraizadas por parcela, após sua transformação em arc $\operatorname{sen} \sqrt{\mathrm{p} / 100}$

\begin{tabular}{lcc}
\hline Causa de Variação & G.L. & F \\
\hline Espéeies (E) & 3 & $63,71^{* *}$ \\
Doses (D) & 2 & $95,51^{* *}$ \\
Interação (E x D) & 6 & $34,68^{* *}$ \\
\hline (Tratamentos) & $(11)$ & - \\
Blocos & 3 & 0,49 \\
Resíduo & 33 & - \\
\hline Total & 47 & - \\
$* *$ Significativo ao nível de $1 \%$ de probabilidade &
\end{tabular}


Como a interação Espécies $\mathrm{x}$ Doses foi significativa, efetuou-se um desdobramento dos graus de liberdade, estudando-se o comportamento das três doses dentro de cada espécie. 0 resultado encontra-se no quadro 3.

Quadro 3 - Análise de variância do número de estacas enraizadas por parcela, com desdobramento dos graus de liberdade.

\begin{tabular}{lcc}
\hline Causa deVariação & G.L. & F \\
\hline Espécies & 3 & $63,71^{* *}$ \\
Doses dentro E1 & 2 & $17,04^{* *}$ \\
Doses dentro E2 & 2 & $114,51^{* *}$ \\
Doses dentro E3 & 2 & $22,29^{* *}$ \\
Doses dentro E4 & 2 & $45,71^{* *}$ \\
\hline (Tratamentos) & $(11)$ & - \\
Blocos & 3 & 0,49 \\
Residuo & 33 & - \\
\hline Total & 47 & -
\end{tabular}

* * Significativo 20 nivel de $1 \%$ de probabilidade.

As médias de cada espécie foram:

niE1 $=31,26 \mathrm{a}$

$\hat{\mathrm{mE}} 2=47,36 \mathrm{~b}$

$\hat{\mathrm{mE}} 3=18.76 \mathrm{c}$

$\hat{\mathrm{mE}} 4=18,66 \mathrm{c}$

Duas médias com a mesma letra não diferem entre si. Tukey $\triangle 5 \%=6,53$, para comparar as médias de duas espécies.

As médias das doses dentro de cada espécie foram:

\begin{tabular}{l|c|c|r|r}
\hline \multirow{2}{*}{ Doses } & \multicolumn{4}{|c}{ Espécies } \\
\cline { 2 - 5 } & E1 & E2 & E3 & E4 \\
\hline D1 & $19,79 \mathrm{a}$ & $14,65 \mathrm{a}$ & $4,36 \mathrm{a}$ & $15,00 \mathrm{a}$ \\
D2 & $44,03 \mathrm{~b}$ & $49,83 \mathrm{~b}$ & $32,14 \mathrm{~b}$ & $0,81 \mathrm{~b}$ \\
D3 & $29,97 \mathrm{a}$ & $77,61 \mathrm{c}$ & $19,79 \mathrm{c}$ & $40,17 \mathrm{c}$ \\
\hline
\end{tabular}


Dentro de cada espécie, duas médias com a mesma letra não diferem entre si. Tukey $\triangle 5 \%=10,27$, para comparar duas médias de doses dentro de uma espécie.

O coeficiente de variação do experimento referente ao número de estacas enraizadas foi da ordem de $20,34 \%$.

Houve uma diferença significativa ao nivel de $1 \%$ de probabilidade para as espécies e para as doses; sendo que ocorreu ainda interação significativa entre espécies e doses.

A comparação das médias de espécies pelo teste de Tukey mostrou que somente não existe diferença significativa entre as espécies Bougainvillea spectabilis e Thuya occidentalis. Pela ordem, os melhores resultados foram obtidos com as espécies, Cupressus sempervirens, Rhododendron Simsii e finalmente Bougainvillea spectabilis ou Thuya occidentalis, indiferentemente. Para Rhododendron, a melhor dosagem do regulador de crescimento foi de $10 \mathrm{ml} / 1$, que diferiu das doses $O$ e $20 \mathrm{ml} / 1$, sendo que estas não diferiram entre si. Para Cupressus, Bougainvillea e Thuya, as três dosagens diferiram entre si; para Cupressus a melhor dosagem foi de $20 \mathrm{ml} / \mathrm{l}$; para Bougainvillea a melhor foi de $10 \mathrm{ml} / \mathrm{l}$; e no caso de Thuya a melhor dosagem revelou ser de $20 \mathrm{ml} / \mathrm{l}$.

No que se refere ao peso fresco médio das raizes (em gramas) por parcela, os resultados obtidos encontram-se no quadro 4.

Quadro 4 - Peso fresco médio das raizes (g) por parcela.

\begin{tabular}{l|c|c|c|c}
\hline Tratamentos & 19 Bloco & 29 Bloco & 39 Bloco & 49 Bloco \\
\hline E1D1 & 1,8 & 1,9 & 2,0 & 1,6 \\
E1D2 & 2,5 & 2,4 & 3,0 & 2,9 \\
E1D3 & 4,3 & 4,4 & 4,0 & 4,8 \\
E2D1 & 1,6 & 1,4 & 0,0 & 1,6 \\
E2D2 & 4,3 & 4,5 & 4,5 & 4,0 \\
E2D3 & 4,9 & 5,2 & 5,3 & 5,0 \\
E3D1 & 1,4 & 0,0 & 0,0 & 0,0 \\
E3D2 & 4,0 & 3,8 & 3,7 & 3,8 \\
E3D3 & 3,8 & 3,9 & 3,8 & 3,6 \\
E4D1 & 1,6 & 1,8 & 1,8 & 1,7 \\
E4D2 & 0,0 & 0,0 & 0,0 & 0,0 \\
E4D3 & 3,2 & 3,6 & 3,4 & 3,3 \\
\hline
\end{tabular}

A análise de variância do peso fresco médio das raizes, encontra-se no quadro 5. 
Quadro 5 - Análise de variância do peso fresco médio das raizes (g) por parcela.

\begin{tabular}{lcc}
\hline Causa de Variação & G.L. & F \\
\hline Espécies (E) & 3 & $53,92^{* *}$ \\
Doses (D) & 2 & $257,69 *$ \\
Interação (E x D) & 6 & $46,15^{* *}$ \\
\hline (Tratamentos) & $(1$ r) & - \\
Blocos & 3 & 0,38 \\
Resíduo & 33 & - \\
\hline Total & 47 & - \\
$* *$ Significativo ao nivel de $1 \%$ de probabilidade. &
\end{tabular}

Para estudo do comportamento das três doses dentro de cada espécie, efetuou-se a análise com desdobramento dos graus de liberdade da interação Espécies x Doses (quadro 6).

Quadro 6 - Análise de variância do peso fresco médio das raizes (g) por parcela, com desdobramento dos graus de liberdade.

\begin{tabular}{lcc}
\hline Causa de Variação & G.L. & F \\
\hline Espécies & 3 & $53,92^{* *}$ \\
Doses dentro E1 & 2 & $51,69^{*}$ \\
Doses dentro E2 & 2 & $134,77^{*}$ \\
Doses dentro E3 & 2 & $122,15^{*}$ \\
Doses dentro E4 & 2 & $87,62^{* *}$ \\
\hline (Tratamentos) & $(11)$ & - \\
Blocos & 3 & 0,38 \\
Residuo & 33 & - \\
\hline Total & 47 & - \\
* Significativo ao nivel de $1 \%$. & &
\end{tabular}


As médias de cada espécie foram:

$$
\begin{aligned}
& \hat{\mathrm{mE} 1}=2,97 \mathrm{~b} \\
& \hat{\mathrm{mE}} 2=3,52 \mathrm{a} \\
& \hat{\mathrm{nE}} 3=2,65 \mathrm{~b} \\
& \hat{\mathrm{mE}} 4=1,70 \mathrm{c}
\end{aligned}
$$

Duas médias com a mesma letra não diferem entre si. Tukey $\triangle 5 \%=0,40$, para comparar as médias de duas espécies.

As médias das doses dentro de cada espécie foram:

\begin{tabular}{c|c|c|c|c}
\hline \multirow{2}{*}{ Doses } & \multicolumn{4}{|c}{ Espécies } \\
\cline { 2 - 5 } & $\mathrm{E} 1$ & $\mathrm{E} 2$ & $\mathrm{E} 3$ & $\mathrm{E} 4$ \\
\hline D1 & $1,82 \mathrm{a}$ & $1,15 \mathrm{a}$ & $0,35 \mathrm{a}$ & $1,72 \mathrm{a}$ \\
D2 & $2,70 \mathrm{~b}$ & $4,32 \mathrm{~b}$ & $3,82 \mathrm{~b}$ & $0,00 \mathrm{~b}$ \\
D3 & $4,38 \mathrm{c}$ & $5,10 \mathrm{c}$ & $3,78 \mathrm{~b}$ & $3,38 \mathrm{c}$ \\
\hline
\end{tabular}

Dentro de cada espécie, duas médias com a mesma letra não diferem entre si. Tukey $\triangle 5 \%=0,63$, para comparar duas médias de doses dentro de uma mesma espécie.

O coeficiente de variação do experimento referente ao peso fresco médio das raízes foi da ordem de $13,28 \%$.

Houve uma diferença significativa ao nível de $1 \%$ de probabilidade para as espécies e para as doses; sendo que verificou-se ainda interação significativa entre espécies e doses.

A comparação das médias de espécies pelo teste de Tukey mostrou que somente não existe diferença significativa entre as espécies ornamentais Rodhodendron Simsii e Bougainvillea espectabilis. Pela ordem, os melhores resultados foram obtidos com as espécies, Cupressus sempervirens, Rhododendron Simsii ou Bougainvillea spectabilis e finalmente Thuya occidentalis. Para Rhododendron, Cupressus e Thuya, as três doses diferiram entre si; sendo que a melhor dosagem para estas espécies revelou ser a de $20 \mathrm{ml} / 1$ de Exubérone. Para Bougainvillea mostraram-se melhores as doses 10 ou $20 \mathrm{ml} / 1$, que não diferiram entre si, mas revelaram-se significativamente distintas de $0 \mathrm{ml} / \mathrm{li}$ tro de água. 


\section{CONCLUSOES}

Os resultados obtidos neste ensaio permitem as seguintes conclusōes:

1. Pela análise do número de estacas enraizadas e do peso fresco médio das raizes, verificou-se que, pela ordem, os melhores resultados da aplicação do regulador de crescimento, foram obtidos com Cupressus sempervirens, Rhododendron Simsii, Bougainvillea spectabilis, e finalmente Thuya occidentalis.

2. Para Cupressus sempervirens e Thuya occidentalis constatou-se que a dosagem de $20 \mathrm{ml}$ de Exubérone por litro de água, é a mais adequada para o método de tratamento lento das estacas.

3. Para Rhododendron Simsii e Bougainvillea spectabilis observou-se que as dosagens de 10 ou $20 \mathrm{ml}$ de Exubérone por litro de água revelaram-se adequadas para o método lento de tratamento das estacas a serem enraizadas.

\section{SUMMARY}

\section{ACTION OF A GROWTH REGULATOR ON ROOTING OF CUTTINGS OF FOUR ORNAMENTAL PLANTS.}

Cuttings from secondary branches of Rhododendron Simsii, Cupressus sempervirens, Bougainvillea spectabilis and Thuya occidentalis were collected in October in Jaboticabal, São Paulo. Following treatment with the rootpromoting compound, Exubérone, at 0,10 , and $20 \mathrm{ml} / 1$, the cuttings were planted and observed in order to determine the number of stems rooted and the average fresh weight of the roots. The results obtained after 80 days permit the conclusion that the different ornamental species responded differently to a given concentration of Exubérone. The rooting response, in order of decreasing effectiveness, was as fallows: Cupressus sempervirens (20 $\mathrm{ml} / 1$ ), Rhododendron Silmsii (10 or $20 \mathrm{ml} / 1$ ), Bougainvillea spectabilis (10 or $20 \mathrm{ml} / \mathrm{l})$, and finaly Thuya occidentalis $(20 \mathrm{ml} / 1)$.

\section{LITERATURA CITADA}

ARENS, T. \& K. ARENS 1972 - O enraizamento de Eucalyptus no clima do Estado de São Paulo. Ciência e Cultura 24 (3) : 233-278.

BRANDI, R. M. \& N. F. BARROS 1971 - Enraizamento de estacas de Pinus caribaea var. hondurenses. Rev. Ceres 18 (98): 270-278.

COOPER, W. C. 1935 - Hormones in relation to root formation on stem cuttings. Plant Physiol. 10: 789-794.

FISCHNICH, O. 1935 - Ủber den einfluss von B-indolylessigsäure auf die blattbewegungen und die adventvwurzelbildung von Coleus. Planta 24 : 552-583.

GOMES, F. P. 1970 - Curso de Estatística Experimental. Livraria Nobel S. A. São Paulo, Brasil. 284 pp.

KRAMER, P. J. \& T. T. KOZLOWSKI, 1960 - Physiology of Trees. McGraw-Hill Book Company. New York, U. S. A. 642 pp. 
RÚBIA, A. C., R. INFORZATO \& C. P. ABREU 1965 - Efeito de hormônios vegetais sobre o enraizamento de estacas de amoreira, em posição normal e invertida. Bragantia 24 (11): 125-131.

SNEDECOR, G. W. 1966 - Metodos Estadísticos Aplicados a la Investigacion Agricola y Biologica. Compañia Editorial Continental ,S. A. Mexico. 626 pp.

SNYDER, W. E. 1954 - The rooting of leafy stem cuttings. Natl. Hort. Mag. 33: 1-18.

STEEL, R. G. \& J. H. TORRIE $1960-$ Principles and Procedures of Statistics. McGraw-Hill Book Company. New York, U. S. A. 481 pp.

THIMANN, K. V. \& J. BEHNKE 1950 - The use of auxines in the rooting of wood cuttings. Maria Moors Cabot Found. Publ. 1, Harvard Forest, Petershan, U.S.A.

ZIMMERMAN, P. W. \& F. WILCOXON 1935 - Several chemical growth substances which cause initiation of roots and other responses in plants. Contrib. Boyce Thompson Inst. 7: 209-229.

ZIMMERMAN, P. W., W. CROCKER \& HITCHCOCK 1933 - Initiation and stimulation of roots from exposure of plants to carbon monoxide gas. Contrib. Boyce Thompson Inst. 2: 205-219. 